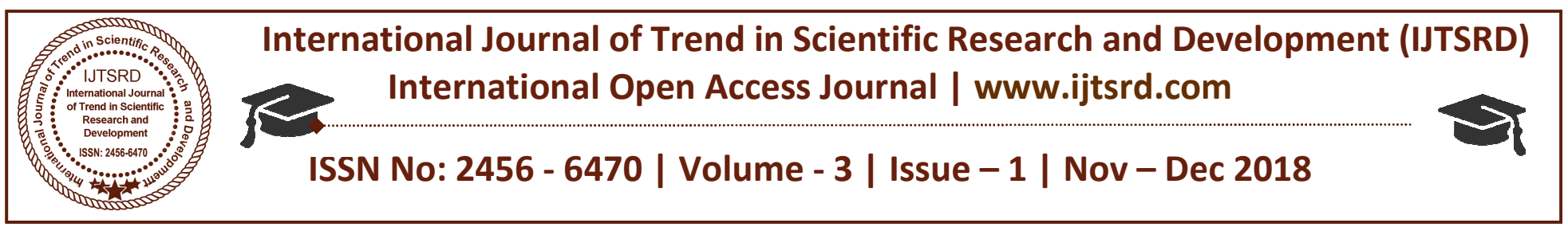

\title{
Comparison of Infection Episodes in CKD Patients with or without Hemodialysis from Tribal Population
}

\author{
Punit Gupta, Swati Sharma, Ashish Deo \\ Nephrology Unit, Department of Medicine, Dr. Bhimrao Ambedkar Hospital and \\ Pt. Jawaharlal Nehru Memorial Medical College, Raipur, Chhattisgarh, India
}

\begin{abstract}
Chronic kidney diseases (CKD) is a progressive and irreversible deterioration of renal function. Patients with CKD are prone to a variety of infections. Further chronic hemodialysis increases the infections and related morbidity and mortality. The present study was conducted to assess the probability of infection episode in CKD patients in patients with or without haemodialysis. A Cross sectional observational study was conducted with a total 56 patients with CKD. Clinical and biochemical data related to infections were collected from the individual patient records. The results showed that the chills and rigors, increased TLC, and elevated ESR were found to more in CKD patients on chronic haemodialysis. Further, our results suggested that CKD patient population showed increased-risk for the development of lethal sepsis. Hence, identification of the causes of infection and the appropriate treatment based on the severity of symptoms are essential for CKD patients who are on dialysis.
\end{abstract}

Keywords: Chronic kidney disease, Infection, ESRD, Dialysis, Sepsis.

\section{INTRODUCTION:}

Chronic kidney diseases (CKD) is a progressive and irreversible deterioration of renal function due to slow destruction of renal parenchyma, eventually leading to death when sufficient number of nephrons have been damaged (1). CKD is increasingly recognized as a global public health problem (2). CKD is more prevalent in the elderly population. However, while younger patients with CKD typically experience progressive loss of kidney function, $30 \%$ of patients over 65 years of age with CKD have stable disease (3).
Patients with CKD are prone to a variety of infections. Infection results in increased mortality and morbidity among patients in pre dialysis stages, in which debility due to uremic state increases the risk of infection (4). The risk can be nullify by prescription of hemodialysis to reduce uremic condition but it further predisposes other culprit that leads to occurrence of infection (5). Source of Infection can be due compromised immunity of patients due underlying multisystem diseases. Infectious disease is the second leading cause of death among dialysis patients, and it is generally assumed that the mortality rate of infectious disease is considerably higher in dialysis patients than in the general population (6). In particular, the mortality rate of sepsis contributed to $69.5 \%$ of the difference in infectious disease mortality between dialysis patients and the general population. Infection-related hospitalizations contribute substantially to excess morbidity and mortality in patients with end-stage renal disease (ESRD). As infection is a primary issue when caring for patients who receive maintenance dialysis it is very much important to understand the infection in CKD patients. The present study was conducted to assess the probability of infection episode in CKD patients in patients with or without haemodialysis.

\section{Materials and Methods}

A Cross sectional observational study was conducted in the nephrology unit, Department of Medicine, Dr. Bhimrao Ambedkar Hospital Raipur (C.G.) For this study a total 56 patients with CKD were recruited during February to March, 2018. Institutional ethical committee of the Pt. J.N.M. Medical College Raipur has approved the study protocol. All patients provided the written informed consent before participating in the study. An approved pre-structured tools or format 
was used to collect the information that include basic parameters such as age, sex, diabetes millitus, hemoglobin, complete blood count, CRP, Urea, Creatinine, sodium, potassium, urine routine, Thyroid, number of dialysis undergone, site of center line for hemodialysis, urine culture, X-RAY Chest and usg abdomen were collected from the individual patient records. In this study increased ESR and TLC $(>11000 / \mathrm{L})$ was considered as an indicator for infection. CKD staging of diseases has been calculated by MDRD formula. Data was collected by using indirect method and was entered and analysed by using Microsoft Excel. The data was presented as number and percentage in each group.

\section{RESULTS}

Baseline characteristics of the study subjects was shown in table 1 . About $82.14 \%$ of the patients were male. The mean age of the study subjects was $41 \pm 15.03$ years. About $98.21 \%$ CKD patients were found to be anaemic ( $\mathrm{Hb}<12 \mathrm{gm})$. Among study subjects only $5.35 \%$ were having DM. Out of total 56 CKD patients included in study, $47(83.2 \%)$ were on haemodialysis and remaining 9 (16.07) were without haemodialysis. Comparison of risk factors of infection between patients with or without haemodialysis were shown in Table 2. Elevated CRP (>40mg/litre) was found in all study subjects. Total leukocyte count $>11000$, was found in $76.6 \%$ and $66.7 \%$ respectively in patients with or without haemodialysis (Figure 1). Extremely elevated ESR count $(>100 \mathrm{~mm} / \mathrm{hr})$ was found only in patients on haemodialysis $(17.0 \%)$. In patients with or without haemodialysis sepsis was found to be $72.3 \%$ and $88.9 \%$ respectively. The history of chills and rigors was found only in patients on haemodialysis (100\%) (Figure2).

\section{Discussion}

Multiple lines of research indicated that acute infections contribute substantially to the high rates of hospitalization and mortality in patients with ESRD (7). Hemodialysis procedure per se as well as disturbances in both innate and adaptive immunity make hemodialysis patients susceptible to infections (8). Episodes of bacteremia account for the majority of severe infections in hemodialysis patients (9). Besides bacterial infections, another common problem in hemodialysis units is the blood transmitted viral infections such as HBV, HCV and HIV (10). In present study the chills and rigors, increased TLC, and elevated ESR were found to more in CKD patients on chronic haemodialysis. Comparison of lymphocyte counts in patients with chronic renal failure treated successively by regular haemodialysis showed highest lymphocyte counts in patients on regular haemodialysis (11). In the present study the infections were higher in the patients on hemodialysis, this might be due to the catheter inserted for haemodialysis in our patient. These results were supported by a study which report threefold higher risk of infection-related death in haemodialysis patients who used a catheter than among those who used an arteriovenous fistula (12).

The results of our study concluded that majority of patients with chronic kidney diseases on long term haemodialysis were more prone to develop infection in campare to patients who were not on haemodialysis. Keeping this fact into consideration better prophylactic steps can be taken to reduce infection related morbidity and mortality in CKD patients undergoing chronic haemodialysis.

\section{Reference}

1. Stringer S, Sharma P, Dutton M, Jesky M, Ng K, Kaur $\mathrm{O}$, et al. The natural history of, and risk factors for, progressive Chronic Kidney Disease (CKD): the Renal Impairment in Secondary care (RIISC) study; rationale and protocol. BMC Nephrology. 2013;14(1):95.

2. Levey A S, Atkins R, Coresh J, Cohen E P, Collins A J, Eckardt K U, et al. Chronic kidney disease as a global public health problem: approaches and initiatives - a position statement from Kidney Disease Improving Global Outcomes. Kidney international. 2007;72(3):24759.

3. Wlodarczyk E, Wlodarczyk Z, Paczek L, Szymanska A, Glyda M, Adamowicz A, et al. Holistic Long-Term Care Over Elderly Kidney Transplant Recipients. Transplantation proceedings. 2018;50(6):1900-3.

4. Wang H E, Gamboa C, Warnock D G, Muntner P. Chronic kidney disease and risk of death from infection. American journal of nephrology. 2011;34(4):330-6.

5. Minnaganti V R, Cunha B A. Infections associated with uremia and dialysis. Infectious disease clinics of North America. 2001;15(2):385406, viii.

6. Berman S J, Johnson E W, Nakatsu C, Alkan M, Chen R, LeDuc J. Burden of Infection in Patients 
International Journal of Trend in Scientific Research and Development (IJTSRD) ISSN: 2456-6470

with End-Stage Renal Disease Requiring LongTerm Dialysis. Clinical Infectious Diseases. 2004;39(12):1747-53.

7. Dalrymple L S, Go A S. Epidemiology of acute infections among patients with chronic kidney disease. Clinical journal of the American Society of Nephrology : CJASN. 2008;3(5):1487-93.

8. Eleftheriadis T, Kartsios C, Yiannaki E, Kazila P, Antoniadi G, Liakopoulos V, et al. Chronic inflammation and CD16+ natural killer cell zetachain downregulation in hemodialysis patients. Blood purification. 2008;26(4):317-21.

9. Sarnak M J, Jaber B L. Mortality caused by sepsis in patients with end-stage renal disease compared with the general population. Kidney international. 2000;58(4):1758-64.

10. Eleftheriadis T, Liakopoulos V, Leivaditis K, Antoniadi G, Stefanidis I. Infections in hemodialysis: a concise review - Part 1: bacteremia and respiratory infections. Hippokratia. 2011;15(1):12-7.

11. Papadimitriou M, Baker L R, Seitanidis B, Sevitt L H, Kulatilake A E. White blood count in patients on regular haemodialysis. British medical journal. 1969;4(5675):67-9.

12. Pastan S, Soucie J M, McClellan W M. Vascular access and increased risk of death among hemodialysis patients. Kidney international. 2002;62(2):620-6.

Table 1: Baselinen data of study subjects

\begin{tabular}{|c|c|c|c|c|}
\hline \multirow{3}{*}{\multicolumn{2}{|c|}{$\begin{array}{l}\text { Parameter } \\
\text { CKD Patients }\end{array}$}} & \multirow{2}{*}{ variables } & Number & Percent \\
\hline & & & 47 & $83.92 \%$ \\
\hline & & Without haemodialysis & 9 & $16.07 \%$ \\
\hline & & Male & 46 & $82.14 \%$ \\
\hline & & Female & 10 & $17.85 \%$ \\
\hline 60 & & $>40$ years & 30 & $53.57 \%$ \\
\hline Age group & & $<40$ years & 26 & $46.4 \%$ \\
\hline Diabetic amono CKD & Ire & SCIEnIDM & 3 & $5.35 \%$ \\
\hline Diabetic among C ND & & Non-DM & 53 & $94.64 \%$ \\
\hline Anemi among CKD & & का $<12 \mathrm{gm} \%$ & 55 & $98.21 \%$ \\
\hline Amema among CND & De & menen $>12 \mathrm{gm} \%$ & 1 & $1.78 \%$ \\
\hline & & Right jugular & 18 & $38.29 \%$ \\
\hline Site of insertion of catheter among & KD with HD & Right femoral & 25 & $53.19 \%$ \\
\hline & & AV fistula & & $8.51 \%$ \\
\hline
\end{tabular}

Table 2: Risk for infection among CKD patients

\begin{tabular}{|c|c|c|}
\hline & On HD patients $\mathrm{n}(\%)$ & Without HD patients n(\%) \\
\hline CRP(>40mg/litre) & $47(100)$ & $9(100)$ \\
\hline TLC $>11000$ & $36(76.6)$ & $6(66.7)$ \\
\hline Extremely elevated ESR count $(>100 \mathrm{~mm} / \mathrm{hr})$ & $8(17.0)$ & $0(0)$ \\
\hline Sepsis & $34(72.3)$ & $8(88.9)$ \\
\hline Episodes of chills and rigors & $47(100)$ & $0(0)$ \\
\hline
\end{tabular}

Figure 1: Comparison of Sepsis, episodes of chills and rigors in CKD patients

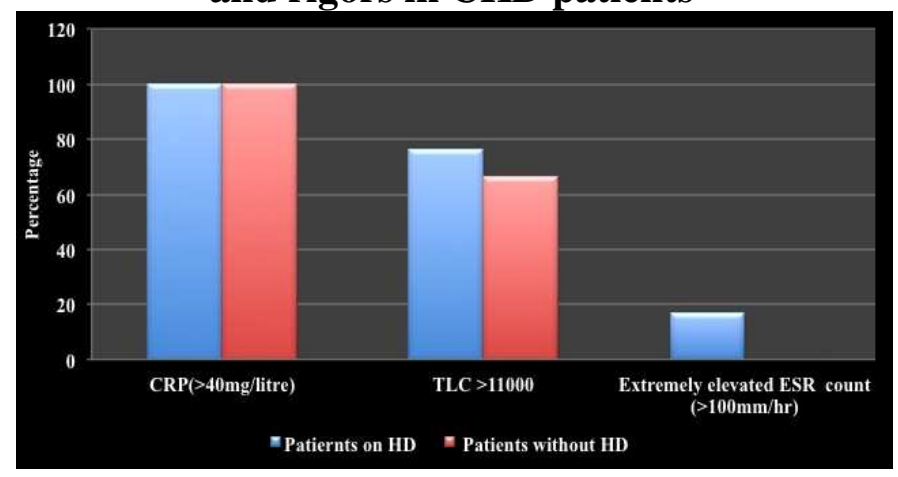

Figure 2: Comparison of abnormal CRP, TLC and ESR in CKD patients

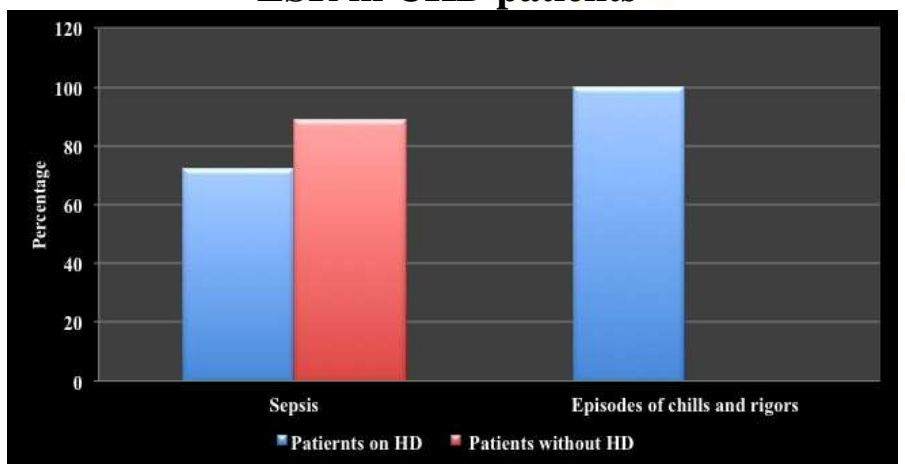

